\title{
The Science Potential of Far-IR/Sub-mm Interferometry and Concepts for the SPIRIT and SPECS Missions
}

\author{
David Leisawitz \\ NASA Goddard Space Flight Center, Greenbelt, MD 20771, USA \\ and the Far-IR Interferometry Mission Study Working Group ${ }^{1}$
}

The FIR/SMM region is unique in the electromagnetic spectrum in its potential for vast increases in sensitivity and angular resolution, and, as a result, information vital to our understanding of the evolution of structure in the universe. About half of the luminosity in the universe is emitted in the far infrared. Evidence for this can be found both in the spectra of individual galaxies (Trentham et al. 1999) and in the cosmic FIR/SMM background found by COBE (Hauser et al. 1998; Fixsen et al. 1998; Dwek et al. 1998). JCMT/SCUBA observations suggest that "a large population of luminous, strongly obscured sources at redshifts $\underset{\sim}{\leqslant}$ is missing from optical surveys" and could account for the background radiation (Blain et al. 1999). Future FIR/SMM measurements of these sources have the potential to reveal the luminosity history of the universe and will provide insight into the processes of galaxy and star formation and galaxy evolution.

Well understood mechanisms give rise to rest-frame FIR emission. A plethora of important interstellar cooling and diagnostic lines are superimposed on a thermal continuum produced by dust emission. The [C II] $158 \mu \mathrm{m}$ line, the brightest line in the spectrum of our galaxy, comes from UV-illuminated molecular clouds and provides a robust measure of star formation activity. FIR $\mathrm{H}_{2} \mathrm{O}$ and $\mathrm{CO}$ lines serve as coolants and bear valuable information about the conditions in collapsing clouds (Harwit et al. 1998; Neufeld 1998). Mid-IR neon line emission can be used to distinguish thermal from nonthermal emissions (Voit 1992). FIR spectral line observations are also needed to measure redshifts of optically obscured sources. Line emission maps made with sufficiently high resolution would allow us to study the dynamics of gas within distant galaxies and in merging and interacting systems.

Comparing the Hubble Deep Field with the corresponding JCMT/SCUBA observation one is struck by the need for better FIR/SMM resolution and the high probability that such an improvement will pay off enormously. Despite its respectable dish diameter (JCMT is $15 \mathrm{~m}$ ), difficulties with source identification

\footnotetext{
${ }^{1}$ Working group members: R. Burg (GSFC), W. Danchi (GSFC), M. Dragovan (JPL), E. Dwek (GSFC), L. Feinberg (GSFC), M. Gaidis (JPL), D. Gezari (GSFC), D.E. Jennings (GSFC), W. Langer (JPL), P. Lawson (JPL), C. Lawrence (JPL), D. Leisawitz (GSFC), J.C. Mather (GSFC), S.H. Moseley, Jr. (GSFC), L.G. Mundy (UMD), R. Mushotzky (GSFC), D. Neufeld (JHU), J. Pedelty (GSFC), M. Shao (JPL), R. Silverberg (GSFC), D. Spergel (Princeton), J. Staguhn (Raytheon/GSFC), M. Swain (JPL), E.L. Wright (UCLA), H.W. Yorke (JPL), and $\mathrm{X}$. Zhang (Raytheon/GSFC)
} 
have hampered interpretation of the SCUBA image. Better sensitivity can be achieved with much smaller cold mirrors in space, and the desired resolution obtained with long interferometric baselines.

We have proposed two FIR/SMM interferometry missions: the Space Infrared Interferometric Telescope (SPIRIT) and the Submillimeter Probe of the Evolution of Cosmic Structure (Mather et al. 2000, Leisawitz et al. 2000). SPIRIT and SPECS are Michelson interferometers that densely sample the u$\mathrm{v}$ plane to provide high quality images and simultaneously yield spectroscopic data. They will be enabled by next-generation technology (Shao et al. 2000). The main characteristics of these instruments are summarized in the table below. Built on a deployable boom and launched in about a decade, SPIRIT would provide baselines extending up to $\sim 30-50 \mathrm{~m}$. This would enable us to resolve the cosmic IR background into discrete sources and make the spectroscopic measurements needed to characterize them. Later SPECS will use separated spacecraft to attain baseline lengths ranging up to $1 \mathrm{~km}$. A natural complement to NGST and ALMA, SPECS would provide a definitive observational basis for understanding the history of and the processes that drive the development of complex structure from the homogeneous early universe.

\begin{tabular}{|l|l|l|}
\hline Parameter & SPIRIT & SPECS \\
\hline Wavelength range & $40-500 \mu \mathrm{m}$ & $40-500 \mu \mathrm{m}$ \\
Spectral resolution & up to $10^{4}$ & up to $10^{4}$ \\
Maximum baseline, $b_{\text {max }}$ & $\sim 30-50 \mathrm{~m}$ & $1 \mathrm{~km}$ \\
Number of collecting mirrors & 2 & 3 \\
Mirror diameter & $3 \mathrm{~m}$ & $4 \mathrm{~m}$ \\
Angular resolution, $\lambda / b_{\max }$ & $2.1^{\prime \prime}$ for $300 \mu \mathrm{m}, 30 \mathrm{~m}$ & $0.06^{\prime \prime}$ for $300 \mu \mathrm{m}, 1 \mathrm{~km}$ \\
Field of view & $3.4^{\prime}$ & $3.4^{\prime}$ \\
Resolution elements & $100 \times 100$ at $300 \mu \mathrm{m}$ & $3,400 \times 3,400$ at $300 \mu \mathrm{m}$ \\
Typical exposure time & $3 \times 10^{4} \mathrm{~s}$ & $3 \times 10^{5} \mathrm{~s}$ \\
Typical sensitivity, $\nu S_{\nu}(1 \sigma)$ & \\
At $\lambda / \Delta \lambda=1,000$ & $2-5 \times 10^{-18} \mathrm{~W} / \mathrm{m}^{2}$ & $0.5-2 \times 10^{-18} \mathrm{~W} / \mathrm{m}^{2}$ \\
At $\lambda / \Delta \lambda=3$ (SED mode) & $0.3-2 \times 10^{-19} \mathrm{~W} / \mathrm{m}^{2}$ & $0.3-1 \times 10^{-19} \mathrm{~W} / \mathrm{m}^{2}$ \\
\hline
\end{tabular}

\section{References}

Blain, A.W., Smail, I., Ivison, R.J. \& Kneib, J.-P. 1999, MNRAS, 302, 632.

Dwek, E., et al. 1998, ApJ, 508, 106

Fixsen, D. J., et al. 1998, ApJ, 508, 123

Harwit, M., Neufeld, D. A., Melnick, G. J. \& Kaufman, M. 1998, ApJ, 497, L105

Hauser, M. G., et al. 1998, ApJ, 508, 25

Leisawitz, D., et al. 2000, in Proc. SPIE, 4013, 36

Mather, J. C., et al. 2000, Rev. Sci. Instr., submitted (astro-ph/9812454)

Neufeld, D. A. 1998, in "After the Dark Ages: When Galaxies Were Young," ed. S. S. Holt \& E. P. Smith, AIP Conf. Proc., 470, 414

Shao, M., et al. 2000, in Proc. SPIE, 4006, 772

Trentham, N., Kormendy, J. \& Sanders, D. B. 1999, AJ, 117, 2152

Voit, M. 1992, ApJ, 339, 495 